OLYMPIA AKTUELL - Aktuelle Olympische Fragestellungen und Entwicklungen 


\title{
Die Autonomie des Sports im Widerstreit: Sportpolitische Herausforderungen der Olympischen Bewegung und der Olympischen Spiele im 21. Jahrhundert
}

\author{
Jürgen Mittag
}

\section{Abstract}

Die Autonomie des Sports gilt als zentrales Strukturprinzip des organisierten Sports. Die Olympische Bewegung und namentlich das Internationale Olympische Komitee (IOC) haben erhebliche Anstrengungen unternommen, die Autonomie des Sports auch im 21. Jahrhundert nachhaltig zu sichern. Trotz dieser Bemühungen steht die Wahrung der Eigenständigkeit des Sports gegenwärtig vor erheblichen Herausforderungen. Jenseits der Besonderheiten einzelner nationaler Sportsysteme haben in den beiden vergangenen Dekaden vor allem übergeordnete politische Entwicklungen dazu beigetragen, dass die Rolle der Olympischen Bewegung anhaltenden Veränderungen unterliegt und die Autonomie des organisierten Sports dabei sukzessive begrenzt wird. Der vorliegende Beitrag beleuchtet - nach einer knappen einführenden historischen Skizze zum Konzept der Autonomie im Sport - überblicksartig sechs exemplarische Problemfelder, die Herausforderungen für die Autonomie des Sports markieren. Dabei werden sowohl Einflussnahmen von außen als auch Aktivitäten der Sportorganisationen selbst beleuchtet, die Auswirkungen auf die sportliche Autonomie zur Folge haben. Zum ersten Problemkreis zählen staatliche Interventionen bei Nationalen Olympischen Komitees in autoritären Ländern, sportbezogene Reformgesetze in OECD-Staaten und Regelsysteme internationaler bzw. supranationaler Organisationen mit Einfluss auf den Sport. Als Problembereiche, in denen die Aktivitäten der Olympischen Bewegung bzw. des IOC selbst die Autonomie herausfordern, werden im Beitrag die mangelhafte Implementierung von GoodGovernance-Prinzipien behandelt, die Vergabepolitik von Sportgroßereignissen und der Rekurs auf das Konzept der Sportdiplomatie. 


\section{Einleitung}

In den vergangenen Jahrzehnten hat der Sport einen grundlegenden Wandel erfahren. Sportbezogene Aktivitäten und vor allem Sportgroßveranstaltungen wie die Olympischen Spiele sind im 21. Jahrhundert zu gesamtgesellschaftlichen Ereignissen avanciert, die mit großer öffentlicher Resonanz in die unterschiedlichsten Lebenswelten hineinwirken und zugleich den verschiedensten Einflüssen - von Politik über Medien und Kultur bis hin zur Wirtschaft - unterliegen. Die Ursachen für den dynamischen Bedeutungszuwachs des Sports sind dabei ebenso breit gesteckt wie vielfältig: Beigetragen hat hierzu vor allem die Vereinnahmung des Leistungssports durch die Massenmedien seit den 1970er und 80er Jahren bei gleichzeitiger Ausdifferenzierung des Mediensystems mit immer mehr privaten Sendern und einer immer umfassenderen Sportberichterstattung. Erheblichen Einfluss hat auch die Kommerzialisierung des Sports und dessen Inanspruchnahme für Image- und Werbezwecke ausgeübt (Cotterell \& Vöpel, 2020). Diese geht einher mit Inszenierungs- und Eventisierungstendenzen, vor allem bei Großveranstaltungen, sowie einer zunehmend wichtigeren Rolle des Sports und seiner Stars in der Populärkultur. Zu den Ursachen für den Wandel im olympischen Sport gehören schließlich auch die veränderten gesellschaftlichen Rahmenbedingungen einer zunehmend säkularisierten sowie ereignisorientierten Bevölkerung, für die Sport-Megaevents zur „Essenz unserer gesellschaftlichen Kommunikation" gehören (Schümer, 1996, S. 8). Der Sport hat sich mittlerweile zu einem zentralen gesellschaftlichen Ereignis entwickelt, das für eine immer stärker funktional ausdifferenzierte Gesellschaft mit immer diversifizierteren Kommunikationsräumen einen Identifikationskern bietet und somit gleichermaßen Anlass, Ort und Bezugspunkt für gesellschaftliche Kommunikation darstellt (Bette, 1990). Auf die Olympische Bewegung und die Olympischen Spiele haben die zunehmenden Interaktionsprozesse des Sports mit anderen Handlungsfeldern erhebliche Wirkung ausgeübt; das IOC hat die Veränderungen seinerseits aber auch selbst forciert. Eine in ihrer Bedeutung kaum zu überschätzende Folge dieser Veränderungsprozesse der letzten Dekaden zeigt sich darin, dass die Autonomie der Olympischen und sportlichen Bewegung in verstärktem Maße herausgefordert wird und die etablierten sportpolitischen Strukturen infolgedessen vor einem grundlegenden Wandel stehen. Das Ausmaß und die Reichweite dieser Veränderungen wird auch für die Ideale der Olympischen Idee sowie für die sportliche Bildung nicht ohne Folgen bleiben.

Die Herausforderungen der Autonomie des olympischen Sports bilden den Mittelpunkt des vorliegenden Beitrags, der dessen strukturelle Verän- 
derungsprozesse an sechs exemplarischen Problemfeldern beleuchtet und zugleich die absehbaren sportpolitischen Folgen für die Autonomie des Sports hervorhebt. Diese markiert ein grundlegendes Prinzip der Olympischen Bewegung, das traditionell auf dem westlich-liberalen Konzept der Vereinigungsfreiheit beruht (Chappelet, 2010, 2018). Als Leitprinzip grenzt die Autonomie des Sports dessen Selbstorganisation durch Verbände von der politischen, rechtlichen und finanziellen Einflussnahme staatlicher oder kommerzieller Akteure ab. Zugleich impliziert die Autonomie des Sports einen pyramidalen Aufbau der Sportstrukturen und die zentrale Rolle von Spitzenverbänden (Geeraert, Mrkonjic \& Chappelet, 2015). Seitens der Olympischen Bewegung wird die Autonomie des Sports als unverzichtbare Notwendigkeit betrachtet, da diese die Aufrechterhaltung der Werte des Sports sichert, die Integrität der Wettkämpfe garantiert und die Grundprinzipien des Olympismus fördert (IOC, 2008). Der Olympischen Charta zufolge „müssen die Sportorganisationen der Olympischen Bewegung die Rechte und Pflichten der Autonomie haben, insbesondere die Regeln des Sports frei aufzustellen und $\mathrm{zu}$ überwachen, die Form und Leitung seiner Organisationen zu bestimmen“, um so „den Sport mit Kultur und Erziehung"verbinden zu können und eine Lebenssicht zu verfolgen, die „in ausgewogener Ganzheit körperliche, willensmäßige und geistige Fähigkeiten miteinander vereint und überhöht." (Vedder \& Lämmer, 2014, S. 7f).

Obwohl die Olympische Bewegung und namentlich das Internationale Olympische Komitee erhebliche Anstrengungen unternommen haben, die Autonomie des Sports auch im 21. Jahrhundert nachhaltig zu sichern, steht die Wahrung der Eigenständigkeit vor erheblichen Herausforderungen. Jenseits spezifischer Besonderheiten der einzelnen nationalen Sportsysteme (Hallmann \& Petry, 2013) haben in den beiden vergangenen Dekaden übergeordnete politische Entwicklungen dazu beigetragen, dass die Autonomie des Sports wiederholt in Frage gestellt wurde und sich die Olympische Bewegung in einem anhaltenden Abwehrkampf befindet. Diese reichen von staatlicher Einflussnahme auf Nationale Olympische Komitees in autoritären Ländern bis hin zu der Infragestellung der Integrität infolge mangelhafter Implementierung von Good-GovernancePrinzipien. Im Lichte des Konzepts dieses Bandes werden die Hintergründe der jeweiligen Problemfelder zunächst kurz vorgestellt, um auf dieser Grundlage dann aktuelle Debatten und Entwicklungen zu veranschaulichen sowie Perspektiven für die Zukunft zu erörtern. Der Beitrag stützt sich dabei vor allem auf Dokumente und Studien aus dem noch jungen Forschungsfeld der Sportpolitik (Mittag, 2010; Kleinfeld, 2018). 
Das IOC und die Genese der verbandlichen Autonomie im Sport

Die verbandliche Autonomie des olympischen Sports war bereits in der Gründungsphase mittelbar Bestandteil der Strukturen des IOC, da die IOC-Mitglieder nicht den Nationalstaat, aus dem sie stammten, sondern vielmehr die Olympische Bewegung ihres Landes vertraten (Krieger \& Wassong, 2021). Damit agierten die Mitglieder - ohne dass der Begriff Autonomie bereits explizit in den Statuten verankert war - weitgehend unabhängig von nationalen Regierungen und deren Einflussnahme. Selbst der Einfluss der nationalen Verbände blieb zunächst begrenzt. Festgelegt war in den Statuten, dass die Mitglieder über ein freies Mandat verfügten und von ihren Verbänden keine Weisung entgegenzunehmen hatten (International Olympic Committee 1908).

Im Original: „Ils ne peuvent accepter de ces sociétés aucun mandat susceptible de les lier en tant que membres du Comité et d'entraver l'indépendance de leurs votes".

Begünstigt wurde die Unabhängigkeit schließlich auch durch einen hohen Grad an finanzieller Freiheit der in der Regel begüterten Mitglieder.

Im Jahre 1949 wurde der Begriff Autonomie mit Bezug auf die Nationalen Olympischen Komitees erstmals in die Olympische Charta aufgenommen. Nach Paragraph 25 der Charta von 1949 wurde die Anforderung, unabhängig und autonom zu agieren, zu einer Voraussetzung für die Anerkennung als Nationales Olympisches Komitee (NOK) (International Olympic Committee, 1949, Art. 25). Im Jahr 1955 wurde diese Bestimmung dahingehend weiter präzisiert, dass die NOKs völlig unabhängig und autonom sowie von politischem, religiösem oder kommerziellem Einfluss vollständig frei sein müssen (International Olympic Committee 1955, Art. 24).

Im Original: "National Olympic Committees must be completely independent and autonomous and entirely removed from political, religious or commercial influence."

Im Jahr 1958 wurde die Charta dann um Bestimmungen zu Sanktionen ergänzt. NOKs, die dem Prinzip der Autonomie nicht folgten, verloren ihre Anerkennung und das Recht, Teilnehmer zu den Olympischen Spielen zu entsenden. Am Ende der 1970er Jahre wurde erstmals keine weitere Verschärfung der Bestimmungen in der Charta vorgenommen, sondern vielmehr konzediert, dass Nationale Olympische Komitees zur Verfolgung ihrer Ziele mit privaten oder staatlichen Organisationen zusammenarbeiten (International Olympic Committee, 1979).

Im Original: „In pursuing their objectives NOCs may cooperate with private or government organisations“ 
Mit diesem Passus trug man nicht zuletzt den politischen Realitäten Rechnung. In der Ära des Kalten Krieges standen die NOKs der kommunistischen Staaten weitgehend unter der Kontrolle der Warschauer Pakt Staaten, während die westlichen NOKs sich anhaltendem politischem Druck ausgesetzt sahen, den Handlungsempfehlungen der nationalen Regierungen zu folgen.

Die Olympischen Spiele von 1976, 1980 und 1984 dokumentieren diesen Druck am deutlichsten, da sie den Höhepunkt der staatlich induzierten sportbezogenen Boykotte markieren (Lennartz, 2009). Im Jahr 1976 reisten 22 afrikanische Teams aus Montreal, dem Austragungsort der Spiele der XXI. Olympiade, ab, um auf diese Weise gegen die Teilnahme Neuseelands zu protestieren, da im Vorfeld die neuseeländische RugbyMannschaft als Nationalteam im Apartheidstaat Südafrika angetreten war. Acht weitere nationale Teams waren zuvor erst gar nicht angereist. Im Jahr 1980, auf dem Höhepunkt des Kalten Kriegs, erklärten die USA aus Protest gegen den sowjetischen Einmarsch in Afghanistan, nicht an den Spielen in Moskau teilzunehmen. Insgesamt 66 NOKs trafen dieselbe Entscheidung, darunter auch das NOK der Bundesrepublik. Sechzehn der in Moskau anwesenden Teams protestierten gegen den sowjetischen Einmarsch in Afghanistan, in dem sie entweder der Eröffnungsfeier fernblieben oder bei der Eröffnungsfeier keine Nationalflagge zeigten, sondern stattdessen die Olympische Fahne bzw. die Flagge ihres NOKs trugen. Weitere sieben Staaten schickten lediglich einen Fahnenträger, aber keine Athlet"innen zur Eröffnung. Als Reaktion auf den Olympiaboykott von 1980 verzichteten vier Jahre später die Sowjetunion und 13 weitere Staaten auf eine Teilnahme an den Sommerspielen in Los Angeles. Der Boykott der Olympischen Spiele 1988 in Seoul durch Nordkorea, Kuba, Äthiopien und Nicaragua markierte das vorläufige Ende der Boykottaktivitäten von Staaten bzw. NOKs im Zuge des Ost-West-Systemkonflikts.

Zum Ende der 1980er Jahre wurde den NOKs zunächst in den Ausführungsbestimmungen und dann in der Olympischen Charta empfohlen, Finanzierungsquellen $\mathrm{zu}$ suchen, die es ihnen ermöglichen, ihre Autonomie zu wahren. Hierbei wurden ausdrücklich auch staatliche Finanzmittel angeführt. Heute heißt es in der Charta, dass die NOKs zur Erfüllung ihrer Aufgaben mit staatlichen Stellen zusammenarbeiten und dabei auf freundschaftliche Beziehungen setzen mögen (International Olympic Committee, 2020, Art. 27).

Im Original: „In order to fulfil their mission, the NOCs may cooperate with governmental bodies, with which they shall achieve harmonious relations. However, they shall not associate themselves with any activity 
which would be in contradiction with the Olympic Charter. The NOCs may also cooperate with non-governmental bodies."

Die jüngeren Anpassungen der Olympischen Charta verdeutlichen, dass eine Finanzierung des Sports durch staatliche Mittel nahezu unverzichtbar ist. Das IOC setzt aber darauf, diese Förderung nicht zum Einfallstor für Interventionen werden zu lassen. Wie wichtig dem IOC seine Autonomie ist, dokumentiert auch die Rede, die IOC-Präsident Thomas Bach vor der UN-Vollversammlung im November 2013 hielt. Er betonte: „Sport [is] truly the only area $[\ldots]$ which has achieved universal law [...]. But to apply this universal law worldwide, sport has to enjoy responsible autonomy. Politics must respect this sporting autonomy" (Bach, 2013). Ein Jahr später, im November 2014, bestätigte die 69. Vollversammlung der UN diesen Anspruch und erklärte ihren Respekt gegenüber der ,independence and autonomy of sport as well as the mission of the IOC in leading the Olympic Movement“" (United Nations, 2014).

\section{Staatliche Interventionen im Nahen Osten und in BRICS-Staaten}

Nach dem Ende des Kalten Krieges hatte die Olympische Bewegung daraufgesetzt, die Autonomie des Sports fortan wieder stärker zu behaupten und staatlichen Interventionen deutlichere Grenzen aufzuzeigen. Vor allem die NOKs sollten vor staatlicher Einflussnahme geschützt werden (Meier, 2021). Da es jedoch vor allem in den autokratisch regierten Staaten des Nahen Ostens und in den BRICS-Staaten (Brasilien, Russland, Indien, China, Südafrika) zu neuerlichen Formen politischer Einflussnahme kam, sah sich das IOC in der vergangenen Dekade wiederholt veranlasst, die Mitgliedsrechte von einzelnen NOKs - etwa des Komitees des Irak sowie derjenigen von Kuweit, Indien, Russland und Brasilien - zeitweilig zu suspendieren, um mithilfe dieser Drohkulisse den Status quo ante wiederherzustellen.

Das NOK von Kuweit wurde seit 2007 gleich mehrfach vom IOC suspendiert, da die Regierung des Scheichtums erheblichen Einfluss auf die Arbeit und Entscheidungen des NOK nahm. Bis zu den Olympischen Spielen von Rio de Janeiro 2016 blieben die Athlet*innen davon unberührt, angesichts der neuerlichen Suspendierung im Oktober 2015 sahen sich die qualifizierten kuwaitischen Sportler dann aber gezwungen, bei den Spielen in Brasilien als Unabhängige Olympische Atbleten unter olympischer Flagge anzutreten, während zugleich die Rechte des Nationalen Olympischen Komitees ausgesetzt wurden. 
Als der irakische Sportminister im Mai 2008 die Auflösung des NOKs und aller nationalen Sportverbände bekanntgab und die Funktionsträger durch Regierungsvertreter ersetzte, wurde dies von der Regierung mit Korruption und unzulänglichen Wahlprozessen in den Sportorganisationen begründet. Als Reaktion darauf suspendierte das IOC im Juli 2008 das irakische NOK und kündigte an, die Athlet"innen von der Teilnahme an den Spielen in Peking auszuschließen. Erst als die irakische Regierung akzeptierte, bis spätestens Ende November des Jahres eine Neuwahl des Nationalen Olympischen Komitees zuzulassen, wurde der Suspendierungsentschluss aufgehoben und nationalen Athlet*innen des Landes doch noch eine Beteiligung an den Spielen in Peking ermöglicht.

In Indien hatte die Regierung im Dezember 2012 einen Gesetzesentwurf eingebracht, der darauf zielte, Einfluss auf die Zusammensetzung der Indian Olympic Association (IOA) zu nehmen, die als NOK von Indien fungierte. Vorgesehen war, Oligarchisierungstendenzen des indischen olympischen Verbands zu begrenzen und hierzu sowohl eine Amtszeitbegrenzung als auch ein Höchstalter von 70 Jahren für Mitglieder einzuführen. Das Internationale Olympische Komitee betrachtete dieses Gesetzesvorhaben der indischen Regierung jedoch als Verletzung der sportbezogenen bzw. olympischen Autonomie und suspendierte infolgedessen die IOA. Die drei für die Olympischen Winterspiele in Sotschi 2014 qualifizierten indischen Sportler mussten infolgedessen ebenfalls zunächst als sogenannte Unabbängige Olympische Atbleten antreten.

Die weltweit am stärksten beachtete Maßregelung eines NOKs erfolgte in der letzten Dekade im Zuge des russischen Dopingskandals. Das Internationale Olympische Komitee suspendierte Ende 2017 das Nationale Olympische Komitee Russlands (ROC) aufgrund des Dopings bzw. der Fälschung von Dopingproben im Zuge der Olympischen Spiele in Sotschi. Die von der Welt-Anti-Doping-Agentur (WADA) beauftragten Untersuchungen, die sogenannten McLaren-Reports, hatten zuvor jahrelange systematische Dopingmanipulationen, die von staatlicher Seite aus unterstützt bzw. wesentlich veranlasst worden waren, dokumentiert. Die Manipulationen sollten dazu beitragen, dass Russland nach einem enttäuschenden elften Rang in der Nationenwertung der Winterspiele 2010 in Vancouver bei den Spielen 2014 in Sotschi, im eigenen Land, besser abschnitt. Mit illegalen Mitteln gelang dies auch, letztlich wurde der Betrug aber aufgedeckt. Die Suspendierung des ROC erfolgt nach längeren Debatten im Vorfeld der Spiele von Pyeongchang 2018 und hatte zur Folge, dass dort 168 Sportler*innen auf Einladung des IOC als Olympische Athleten aus Russland ohne Flagge und Hymne antraten. Nach den Spielen wurde die Suspendierung wieder aufgehoben. Als im Dezember 2019 die Welt- 
Anti-Doping-Agentur Russlands Sport erneut suspendierte, da der bisherige Betrug weiter geleugnet bzw. Dopingdaten unvermindert manipuliert wurden und zudem die Bereitschaft zu Reformen begrenzt blieb, legte Russland vor dem Internationalen Sportgerichtshof CAS Berufung ein. Das Gericht änderte die zunächst für vier Jahre ausgesprochene Sperre in eine Zweijahressperre. Dieser Zeitraum schloss eine Teilnahme des ROC an den Olympischen Sommerspielen in Tokio und den Winterspielen in Peking aus, führte aber zu erheblicher Kritik, nicht zuletzt auch am IOC, das sich im Umgang mit Russland zu konziliant verhalten habe.

Während die bisher angeführten Suspendierungen maßgeblich als Reaktion des IOC auf staatliche Einflussnahme auf den olympischen Sport zu werten sind, erfolgte die kurzzeitige Suspendierung des NOKs von Brasilien im Jahre 2017 mit Blick auf die Verfehlungen von dessen Präsidenten Carlos Nuzman. Dieser hatte als Organisationschef der Olympischen Spiele 2016 fungiert und war festgenommen worden, da er sich, der Staatsanwaltschaft zufolge, an der Vergabe von Bauaufträgen und Dienstleistungsverträgen im Zuge der Spiele bereichert hatte und zudem rund 1,7 Millionen Euro an Bestechungsgeldern für den Kauf von Stimmen bei der Vergabe der Spiele an Rio organisiert hatte.

Deutlich wird an den angeführten Beispielen, dass die Autonomie des Sports bzw. der NOKs eine beständige Herausforderung für den olympischen Sport markiert. Vor allem wirtschaftlich starke und zugleich autoritär regierte Staaten nutzen den Sport, um damit nationales Prestige zu erzielen. Dabei scheuen sie nicht davor zurück, auch stärkeren Einfluss auf nationale Sportorganisationen und das jeweilige NOK zu nehmen. In einigen Fällen zielen die Interventionen aber auch lediglich darauf, zweifelhaften Praktiken oder Unregelmäßigkeiten im NOK entgegenzuwirken. Für die Olympische Bewegung und namentlich für das IOC bedeutet es einen permanenten Drahtseilakt, die Einflussnahme von Staaten zurückzuweisen, dabei gleichzeitig aber die staatliche finanzielle Förderung nicht grundsätzliche infrage zu stellen.

Nationalstaatliche Gesetze mit Auswirkungen auf den olympischen Sport in OECD-Staaten

Dass die Autonomie des Sports nicht nur in autoritären Ländern oder Staaten mit defizitären demokratischen Strukturen vor Herausforderungen steht, zeigen Beispiele aus Staaten der OECD-Welt.

OECD-Staaten sind Mitglieder in der "Organisation for Economic CoOperation and Development", die vor allem für Demokratie und Markt- 
wirtschaft eintritt. Die OECD ist eine internationale Organisation mit gegenwärtig (2021) 37 Mitgliedstaaten, die in der Regel demokratischen Kriterien Rechnung tragen sowie einen überdurchschnittlichen Entwicklungsstand und ein hohes wirtschaftliches Niveau aufweisen. Das durchschnittliche Bruttoinlandsprodukt (BIP) der OECD-Staaten lag im Jahre 2020 bei 44.625 Dollar.

In Südkorea sind in den vergangenen Jahren im organisierten Sport Zentralisierungsprozesse forciert worden. So wurden zunächst das Koreanische Nationale Olympische Komitee (KOC) und der Korea Sports Council (KSC) im Jahr 2009 zusammengelegt. Im März 2016 kam es dann zu einem neuerlichen Zusammenschluss, bei dem der Korea Council of Sport for All mit dem Koreanischen Nationalen Olympischen Komitee zum Korea Sport \& Olympic Committee (KSOC) fusionierte. Nach diesem Zusammenschluss ist das KSOC sowohl für den Spitzensport als auch für den Breitensport zuständig. In Korea, das mit Pyeongchang den Austragungsort der Olympischen Winterspiele 2018 stellte, agierte das KSOC zeitweilig wie eine quasi-staatliche Organisation. Das KSOC setzte dabei wesentliche Zielvorgaben der nationalen Sportpolitik bzw. des koreanischen Ministeriums für Kultur, Sport und Tourismus (MCST) um. Seitens des IOC wurde die reibungslose Durchführung der Olympischen Winterspiele zwar begrüßt, die Einflussnahme des Staates aber mit zunehmenden Bedenken verfolgt. Als die südkoreanische Regierung im Jahr 2019 dann im Zuge der Berichterstattung über Missbrauchsskandale im koreanischen Sport empfahl, das KSOC wieder in zwei Einheiten aufzuteilen - eine als NOK und die andere zur Beaufsichtigung des Breitensports - meldete sich das IOC kritisch zu Wort. Da seitens der koreanischen Regierung zudem die Entlassung des KSOC-Generalsekretärs Kim Seung-ho gefordert und die Umsetzung der neuen Statuten des KSOC, die zuvor vom IOC genehmigt worden waren, blockiert wurde, erklärte das IOC seine ernste Besorgnis über die Intervention der südkoreanischen Regierung im Sport. Zu Sanktionen ist es in diesem Fall jedoch bis heute nicht gekommen.

Auch gegenüber europäischen Staaten bzw. NOKs in Europa, wie demjenigen von Italien, wurden in jüngerer Vergangenheit deutliche Warnungen des IOC ausgesprochen. In Italien wurden im Herbst 2019 Pläne des Kabinetts von Ministerpräsident Conte bekannt, mit der Gründung eines neuen Sportdachverbands unter der Bezeichnung Sport $e$ Salute (Sport und Gesundheit) die Strukturen des nationalen Sports grundlegend $\mathrm{zu}$ ändern. Unter anderem sollte die neue Organisation das Programm Sport nei Parchi implementieren und in diesem Zuge die Betreuung allgemein zugänglicher öffentlicher Sportanlagen in Parks 
übernehmen. Das IOC reagierte hierauf mit der Ankündigung, dass die Mitgliedschaft des Nationalen Olympischen Komitees (CONI) im IOC ausgesetzt werden könnte. Dies war umso bemerkenswerter, weil Italien kurz zuvor, im Juni 2019, noch zum Austragungsort der XXV. Olympischen Winterspiele im Februar 2026 in Mailand und Cortina d'Ampezzo gekürt wurde. Als Sport e Salute dann aus der Taufe gehoben und direkt dem Finanzministerium unterstellt wurde, übernahm die neue Organisation wie vorgesehen auch Personal und Sportstätten von CONI. Damit verlor das bis dahin autonom agierende NOK erhebliche Ressourcen. Abgewendet wurde die Suspendierung von CONI letztlich nur dadurch, dass die italienische Regierung unter Ministerpräsident Conte in der letzten Sitzung vor ihrem Rücktritt im Februar 2021 ein Dekret verabschiedete, mit dem sie zusicherte, die Unabhängigkeit des Nationalen Olympischen Komitees in Italien zu garantieren.

$\mathrm{Zu}$ einer deutlichen Verschiebung in der traditionellen Abgrenzung zwischen staatlichem Handeln und verbandlich organisiertem Sport kam es im Jahr 2016 in Deutschland in der Dopingbekämpfung. Bereits seit den 1990er Jahren war in Deutschland immer wieder die Forderung nach einem staatlichen Anti-Doping-Gesetz erhoben worden (Haug, 2006). Mit Verweis auf die Autonomie des Sports fand dieses Ansinnen jedoch politisch keine Unterstützung. Im Jahr 2007 wurde dann jedoch der $\$ 6$ des Arzneimittelgesetzes um ein Verbot des Besitzes von „nicht geringen Mengen" dopingfähiger Substanzen ergänzt. Darüber hinaus wurde im selben Jahr das Gesetz zur Verbesserung der Bekämpfung des Dopings im Sport verabschiedet, mit dem Ziel, kriminelle Strukturen im Sport zu bekämpfen, wozu auch eine begrenzte Sanktionierung des Besitzes von Dopingmitteln eingeführt wurde. Mit diesen Schritten setzte ein verstärktes staatliches Engagement in der Dopingbekämpfung ein. Im Jahr 2009 wurde in München die erste Schwerpunktstaatsanwaltschaft für Doping in Deutschland eingerichtet. Eine grundlegende Neupositionierung des Themas der gesetzlichen Regelung in der Anti-Doping-Politik erfolgte dann nach der Bundestagswahl 2013, als der Koalitionsvertrag zwischen CDU/CSU und SPD das Ziel beinhaltete, weitergehende gesetzliche Maßnahmen umzusetzen. Diesem Auftrag folgend wurde 2015 ein Entwurf für ein Gesetz zur Bekämpfung von Doping im Sport eingebracht und - gegen den Widerstand des Deutschen Olympischen Sportbundes (DOSB) - im Bundestag verabschiedet (Risse, 2018). Am 1. Januar 2016 ist dieser Rechtsakt in Kraft getreten. Bis zum Inkrafttreten dieses Gesetzes lag die Sanktionsgewalt für Vergehen beim organisierten Sport; das neue Gesetz stellt nun aber den Erwerb, den Besitz und die Anwendung von Dopingmitteln und -methoden, um sich einen Vorteil in einem 
Wettkampf zu verschaffen, unter Strafe und schreibt damit dem Staat wesentliche Kompetenzen zu. Der damit verbundene Verlust an Autonomie für den organisierten Sport lässt sich nicht auf einen konkreten Dopingskandal im deutschen Sport zurückführen, sondern ist vielmehr Ausdruck eines allgemeinen Wandels in der deutschen Sportpolitik. Aufgrund der Herausforderungen und Probleme im organisierten Sport ändern staatliche Akteure ihre Position und treten für eine stärkere Rolle des Staates ein.

$\mathrm{Zu}$ einer tendenziell ähnlichen Entwicklung ist es in den USA gekommen, wo im November 2020 ein neues Anti-Doping Gesetz, der sogenannte Rodshenkov Act, verabschiedet wurde. Der Rechtsakt ist nach Grigori Rodschenkov benannt, dem früheren Direktor des Moskauer Anti-Doping-Labors, der den Doping-Skandal 2016 in Russland aufgedeckt hatte. Das Gesetz eröffnet den US-amerikanischen Strafverfolgungsbehörden erweiterte Kompetenzen im Kampf gegen Doping und zielt auf Hintermänner bzw. kriminelle Netzwerke ab. Die Ermittler können bei begründetem Verdacht, dass US-amerikanische Akteure involviert sind, nunmehr weltweit ermitteln. Das IOC und die Welt-Anti-Doping-Agentur (WADA) haben sich gegen das Gesetz ausgesprochen. Sie befürchten einen Bedeutungsverlust der WADA und Rückschritte bei der Vereinheitlichung des Rechtsrahmens im Kampf gegen Doping. Darüber hinaus wird das neue Gesetz aber auch als ein weiteres Einfallstor zur Begrenzung der Autonomie des olympischen Sports gesehen, dessen Kompetenzen zur Ermittlung und rechtlichen Sanktionierung damit begrenzt werden.

Die hier angeführten vier Beispiele aus OECD-Staaten zeugen davon, welche erhebliche Dynamik im olympischen Sport gegenwärtig auszumachen ist. Die eingangs skizzierten Veränderungsprozesse haben dazu geführt, dass auch staatliche Akteure verstärkt für den Sport Verantwortung übernehmen. Diese Haltung kommt vor allem deswegen zum Tragen, weil die beträchtliche Förderung des olympischen Sports auch zunehmende Begehrlichkeiten der Politik weckt, über die Verwendung der staatlichen Gelder mitzuentscheiden. Infolgedessen kommt es auch hier zu verstärkter Einflussnahme. Diese fällt noch umfassender aus, wenn den Akteuren des olympischen Sports eine nur begrenzte Reformbereitschaft unterstellt wird oder offenkundige Fehlentwicklungen auszumachen sind, auf die von der Olympischen Bewegung bislang nicht reagiert wurde. 
Einflussnabme durch internationale und supranationale Akteure auf den Sport

Im Zuge der anhaltenden Entgrenzungs- bzw. Globalisierungprozesse der vergangenen Jahrzehnte hat auch der Einfluss von internationalen Akteuren im Sport zugenommen. Diese haben in den vergangenen Dekaden wiederholt ihre grundsätzliche Unterstützung für die bestehenden Sportstrukturen zum Ausdruck gebracht. Diese Unterstützung ist jedoch nicht bedingungslos und unterliegt zudem anhaltenden Veränderungen. Dies wird besonders mit Blick auf staatliche Akteure im internationalen bzw. supranationalen Raum deutlich. So hat etwa aus der UNFamilie die UNESCO in ihrer überarbeiteten Internationalen Charta für Leibeserziehung und Sport von 2015 zwar grundlegende Prinzipien und Werte des Sports in Erinnerung gerufen und auch die Olympische und Paralympische Bewegung als wichtige Akteure explizit benannt, jedoch darauf verzichtet, einen Hinweis auf die Autonomie des Sports aufzunehmen. Die Europäische Kommission hat in ihren verschiedenen Grundlagen- und Strategiepapieren der beiden letzten Dekaden wiederholt betont, dass sie - wie im Weißbuch zum Sport (Europäische Kommission, 2007) - „die Autonomie der Sportorganisationen und der repräsentativen Strukturen (wie Ligen)" anerkenne. Sie hat dieses Bekenntnis aber in der Regel mit Konditionen verbunden, so etwa in dem Dokument zur Entwicklung der europäischen Dimension des Sports vom 18. Januar $2011 \mathrm{mit}$ dem Hinweis, dass die Berücksichtigung von Good-Governance-Kriterien „eine Voraussetzung für die Autonomie und die Selbstregulierung von Sportverbänden" sei (Europäische Kommission, 2011).

Neben entsprechenden politischen Stellungnahmen hat vor allem das supranationale Recht der Europäischen Union Auswirkungen auf die Frage der Autonomie des Sports ausgeübt (Mittag, 2018). Das wohl prägnanteste Beispiel bot dabei in den 1990er Jahren der Europäische Gerichtshof mit dem viel zitierten Bosman-Urteil im Fußball. Die hier zugrunde gelegten Bestimmungen der Grundfreiheiten des EU-Binnenmarkts waren nicht originär auf den Sport gemünzt, übten auf diesen aber eine grundlegende Wirkung aus und änderten die Strukturen des europäischen Mannschaftssports nachhaltig. Deutlich wurde bereits mit dem Bosman-Urteil, dass die Europäische Union selbst in Bereichen wie der Sportpolitik, in denen sie keinerlei exklusive Kompetenz besaß, durch das supranationale Recht derart umfassend Einfluss nehmen konnte, dass auch die Autonomie des Sports herausgefordert wurde. Weitere aus dem Wirtschaftsrecht abgeleitete sportbezogene Urteile der nachfolgenden Jahre untermauerten diese Sichtweise, die zuletzt bei der ISU-Entscheidung der Europäischen Kommission erneut zum Tragen kam (Mittag, 
2019). Dem Olympiasieger im Eisschnelllaufen Mark Tuitert und dem Staffelweltmeister im Shorttrack Niels Kerstholt war es von der Internationalen Eislaufunion (International Skating Union, ISU), dem Dachverband der Eisschnell- und Eiskunstläufer, untersagt worden, an einem lukrativen Show-Wettkampf in Dubai teilzunehmen, da dieser nicht von der ISU autorisiert war. Angesichts drohender Strafen, die bis zu lebenslangen Sperren reichen, wendeten sich beide Athleten im Juni 2014 an die Europäische Kommission und reklamierten, dass entsprechende Sanktionen eine Einschränkung ihrer Berufsfreiheit darstellen würden. Damit war eine Kernfrage des Sports angesprochen, berührt dieses Problemfeld doch im Kern das Verbands- und Veranstaltungsmonopol des organisierten Sports. Nicht zuletzt deswegen hatte sich auch IOC-Präsident Thomas Bach noch am 21. November 2017, unmittelbar im Vorfeld der erwarteten Kommissions-Entscheidung, mit den europäischen Sportministern in Brüssel getroffen, um für das europäische Sportmodell zu werben und auf dessen potenzielle Aushebelung durch das EU-Kartellrecht zu verweisen. Sowohl die Europäische Kommission, die die Internationale Eislauf-Union in einer Stellungnahme im November 2017 aufforderte, ihre Regeln zu ändern, als auch der Europäische Gerichtshof, der im Dezember 2020 urteilte, gaben den Athleten weitgehend Recht. Die ISU hat dem Urteil des Europäischen Gerichtshofs zufolge andere Veranstalter bei der Ausrichtung eines Wettkampfs unzulässig beeinträchtigt und damit gegen das Wettbewerbsrecht der Europäischen Union verstoßen. Damit ist aber ein wesentlicher Bereich der sportverbandlichen Autonomie berührt. Dem Wettbewerbssport liegt ein Pyramidalsystem zugrunde, das von der internationalen über die kontinentale bis zur nationalen bzw. regionalen und kommunalen Ebene hinunterreicht und letztlich auf einem Monopol des Fachverbandes auf der jeweiligen Ebene basiert. Lange Zeit galt dieses System als unumstritten, da so unter anderem einheitliche Regeln und Sicherheitsstandards bei Wettbewerben gewahrt werden. Seitdem im Sport aber nicht nur zunehmend höhere Umsätze generiert werden, sondern sich auch immer mehr Akteure mit unterschiedlichen Interessen engagieren, wird verstärkt moniert, dass die Verbände ihre Veranstaltungshoheit einseitig im Sinne eines Wirtschaftsunternehmens nutzen, um eigene Ansprüche zu sichern.

Sollte nun das ISU-Urteil künftig verstärkt als Präzedenzfall herangezogen werden, kann dieses erhebliche Implikationen für die künftige Organisation des Sports zur Folge haben. Auch wenn die EU-Institutionen bislang die besondere Rolle der Sportverbände und damit auch eine gewisse Autonomie des olympischen Sports anerkannt haben, werden die rechtlichen Rahmenbedingungen des Binnenmarktes nicht grundsätz- 
lich außer Kraft gesetzt. Damit ist aber zumindest die Tür zu einem pluralistischeren System im Sport geöffnet, das seinerseits wiederum die Autonomie des Sports mittelfristig zur Erosion bringen kann.

\section{Good Governance und Integrität}

Als Herausforderung für die Autonomie des Sports haben sich in den vergangenen Dekaden nicht nur Interventionen von außen erwiesen, sondern auch Missstände und Fehlentwicklungen in den Binnenstrukturen der Olympischen Bewegung. Die Kontrastfolie dazu lieferte ein verstärkter Rekurs auf Governance-Konzepte in der Politik, der dazu führte, dass auch im Sport Good Governance zum vielbeachteten Leitbild avancierte (Sobry, 2011; Geeraert, 2013).

\section{Good Governance}

Seit der Jahrhundertwende werden von Medien und Öffentlichkeit mit Good Governance zunehmend höhere normative Erwartungen mit der Organisation des Sports verbunden. Verstanden wird unter Good Governance die Verantwortung von Sportverbänden und Funktionsträger*innen für die Organisation und Integrität des Sports durch die Entwicklung, Umsetzung und Kontrolle von Verhaltensnormen und -regeln.

Den Ansprüchen an Good Governance ist der Sport im Zuge anhaltender Kommerzialisierungsprozesse gerade zum Ende der 1990er Jahre nicht immer gerecht geworden (Emrich \& Gassmann, 2019). Als Sündenfall des internationalen Sports - und zugleich als entscheidende Wegmarke für die Existenz eines strukturellen Problems im olympischen Sport - gilt der Vergabeprozess der Olympischen Winterspiele an Salt Lake City bei der Session des IOC in Budapest im Jahr 1995. Drei Jahre später, noch vor der Ausrichtung der Spiele, wurden zahlreiche Korruptions- und Bestechungsakte von IOC-Mitgliedern im Zuge des Vergabeverfahrens bekannt. Als dann der Schweizer Marc Hodler, Mitglied des IOC-Exekutivkomitees, im Dezember 1999 mit weiteren Vorwürfen an die Öffentlichkeit ging, wurde das IOC in seinen Grundfesten erschüttert. Die Liste der Anschuldigungen reichte von der Anstellung von Familienangehörigen von IOC-Mitgliedern über die Gewährung von Gratiseinkäufen bei WalMart bis hin zu Einladungen zu Luxusreisen nach Las Vegas, Disneyland, zu den Niagara Fällen und zum Yellowstone Nationalpark. Im Zuge der daraufhin eingeleiteten Untersuchungen wurden 14 IOC-Mitglieder 
sanktioniert, sechs sogar auf Lebenszeit ausgeschlossen. Weitere vier waren bereits zuvor zurückgetreten (Mason, Thibault \& Misener, 2006). Als wesentliche Ursache für den Skandal von Salt Lake City wurde einerseits die anhaltende Kommerzialisierung des Sports mit immer höheren Umsätzen und Begehrlichkeiten ausgemacht, andererseits mangelndes Unrechtsbewusstsein und fehlende Kontrollinstanzen. Diese Rahmenbedingungen hatten es dem Bewerbungskomitee von Salt Lake City erlaubt, in unzulässiger Art Einfluss auf die IOC-Mitglieder zu nehmen und so den Zuschlag für die Winterspiele zu erhalten. Das mediale Echo, das auf die Veröffentlichung dieser Fehlentwicklungen folgte, war verheerend. Zu den wiederkehrenden Forderungen gehörte dabei auch, das IOC fortan stärker zu reglementieren.

Das IOC versuchte dem entgegenzuwirken. Es reagierte entschlossen, beauftragte unter Leitung von Richard Pound eine Ad-hoc-Kommission zur Aufarbeitung des Olympia-Skandals um Salt Lake City und leitete einen grundlegenden Reformprozess ein. Bereits im Frühjahr 1999 wurde eine Ethik-Kommission etabliert, die einen Verhaltenskodex für IOCMitglieder entwickelte und diesen fortan auch überwachte. Eine Reformkommission entwickelte unter der Bezeichnung „IOC 2000“ ein Reformpaket, das vor allem Änderungen im Bewerbungsprozess vorschlug, aber auch zahlreiche Strukturreformen empfahl, die in der Folge dann auch umgesetzt wurden.

Das Bewerbungsprozedere ist seitdem in einen zweistufigen Prozess aufgegliedert. In einem ersten Schritt werden von einer vom IOC-Exekutivkomitee bestellten Evaluierungskommission eine Anzahl von Bewerbern anhand von eher technischen Kriterien vorausgewählt. Der eigentliche Vergabebeschluss erfolgt dann in einem zweiten Schritt durch die Session, die Legislative des IOC. In die Statuten des IOC wurde zudem erstmals eine Amtszeitbegrenzung für den IOC-Präsidenten aufgenommen, der nunmehr zunächst für acht Jahre gewählt wird und dann noch einmal für vier Jahre wiedergewählt werden kann. Die IOC-Exekutive wurde von elf auf fünfzehn Mitglieder vergrößert. Eine weitere Änderung betraf die Altersgrenze für neue IOC-Mitglieder, die - nach dem Ende von Übergangsregelungen - auf 70 Jahre festgelegt wurde. Mit der Reform wurde auch die Gesamtzahl der IOC-Mitglieder auf 115 festgelegt und die Repräsentation von einzelnen Akteursgruppen in der Charta verankert. Im IOC sind nun 15 Athletenvertreter*innen, 15 Mitglieder von internationalen Sportfachverbänden und 15 Vertreter"innen von Nationalen Olympischen Komitees vertreten (MacAloon, 2011). Neue Mitglieder werden zwar weiterhin vom IOC selbst gewählt, können aber 
nun auch von den internationalen Sportfachverbänden, den NOKs und der IOC-Athletenkommission vorgeschlagen werden.

Mit diesen Reformakten hat sich das IOC erheblich modernisiert, demokratisiert und auch den Ansprüchen von Good Governance deutlich stärker Rechnung getragen als andere internationale Sportverbände im selben Zeitraum. Dennoch lag manches weiterhin im Argen. Besonders kritisch erwies sich der Umstand, dass der Olympia-Skandal um Salt Lake City seitens des IOC als einmalige Ausnahme behandelt wurde und die in der Olympischen Bewegung vorhandenen Korruptionserscheinungen nicht als grundsätzliches Problem angegangen wurden. Diese Handhabung forcierte anhaltende Kritik in Medien und Öffentlichkeit. Dies umso mehr, als in den folgenden Jahren weitere Korruptionsfälle bekannt wurden. So brachten 2008 verdeckt operierende BBC-Journalisten in Erfahrung, dass einzelne IOC-Mitglieder sich bereit erklärt hatten, bei der Wahl des Austragungsortes für die Olympischen Sommerspiele 2012 rund 15 bis 20 Stimmen aufzubringen. Auch Medienberichte über den illegalen Weiterverkauf von Tickets für die Spiele 2012 in London durch Mitglieder einzelner NOKs konterkarierten die Good-GovernanceAmbitionen. Den wohl stärksten negativen Effekt übten indes die Präsidenten von anderen Sportfachverbänden aus, die zum Zeitpunkt der Vorwürfe zugleich IOC-Mitglieder waren (Krieger, 2018). Der ehemalige FIFA-Präsident Joao Havelange trat wegen der ISL-Affäre der FIFA im Dezember 2011 aus dem IOC zurück, sein Nachfolger Josef Blatter sah sich wegen der FIFA-Skandale um die WM-Vergabe 2018 und 2022 im Juni 2015 zum gleichen Entschluss veranlasst (Mittag \& Nieland, 2016).

Die Vermarktungsagentur International Sport and Leisure (ISL) wurde Mitte der 1980er-Jahre vom damaligen Adidas-Chef Horst Dassler gegründet. Die Schweizer Agentur war eine Tochtergesellschaft der ISMMGruppe, die u.a. mit den Rechten für die Fußball-Weltmeisterschaften handelte.

Im Jahr 2015 musste mit Lamine Diack als IOC-Ehrenmitglied sowie als Präsident der International Athletics Foundation (IAF) eine der umstrittensten Figuren des internationalen Sports zurücktreten.

$\mathrm{Da}$ die verschiedenen Skandale die Integrität und Legitimität des olympischen Sports diskreditierten, ist der Ruf nach der Durchsetzung von Good-Governance-Prinzipien in den internationalen Sportorganisationen seitdem nicht mehr verstummt. Vielmehr wurden entsprechende Ansprüche auch auf nationale und subnationale Sportverbände ausgeweitet. Mittlerweile gilt die Einhaltung von Good-Governance-Standards im Sport sogar als zentraler Indikator für den Grad der künftigen Autonomie des organisierten Sports (Bonollo \& Gilligan, 2009; Europäi- 
sche Kommission, 2011; Expert Group Good Governance, 2013). Nur wenn entsprechende Prinzipien eingehalten werden und ein hohes Maß an Integrität der Sportverbände dokumentiert wird, kann der organisierte Sport nach landläufiger Auffassung beanspruchen, bei der Regulierung und Ausgestaltung des Sports auch künftig weitgehend autonom zu agieren.

Die Nationalstaaten verlassen sich jedoch nicht mehr allein auf die Selbstheilungskräfte des organisierten Sports. Vor allem der FIFA-Skandal 2015 hat dazu beigetragen, dass die Schweiz begann, in der neben dem IOC auch ein Großteil der olympischen Sportfachverbände seinen Sitz hat, das Handeln der Sportverbände stärker zu reglementieren. Hatte die Schweiz bis dahin nur wenig Interesse gezeigt, ihre nationale Rechtsordnung anzupassen, in der bis 2016 private Bestechung nicht als Straftatbestand galt, so führte eine Gesetzänderung dazu, dass ab 1. Juli 2016 die aktive und passive Privatbestechung ins Strafgesetzbuch aufgenommen wurde. Damit erhielten die Schweizer Behörden, namentlich die schweizerische Bundesanwaltschaft, eine Handhabe, gegen illegale Praktiken im Sport stärker vorzugehen. Auch andere Staaten befassen sich mittlerweile mit den betrügerischen Aktivitäten von Sportfunktionären und setzen damit auf ein höheres Maß an Interventionen. So hatte Tsunekazu Takeda, der Präsident des japanischen Olympischen Komitees, im März 2019 seinen Rücktritt erklärt, da die französische Finanzstaatsanwaltschaft Parquet National Financier (PNF) Ermittlungen wegen Geldern aufgenommen hatte, die kurz vor und kurz nach der Olympia-Vergabe an Tokio im Herbst 2013 an eine Beraterfirma geflossen waren. Angesichts dieser Entwicklung ist zu erwarten, dass fehlende Good-Governance-Standards und lediglich begrenzte Selbstreinigungsprozesse im Sport auch in Zukunft einen zentralen Hebel zur staatlichen Einflussnahme darstellen, der erhebliche Auswirkungen auf die Autonomie des Sports haben wird.

\section{Die Vergabe von Sportgroßveranstaltungen}

Zumindest mittelbaren Einfluss auf die Autonomie des olympischen Sports hat die Vergabepolitik bei Sportgroßereignissen in den letzten Jahren ausgeübt. Diese haben traditionell schon immer starkes Interesse hervorgerufen, aber erst mit den eingangs skizzierten Änderungen im Mediensystem seit etwa Mitte der 1980er hat diese Entwicklung jene umfassende Dimension erreicht, die heute allgegenwärtig ist und sich unter anderem darin widerspiegelt, dass mittlerweile für 30 Sekunden Werbung im Rahmen des Superbowl rund fünf Millionen Euro verlangt 
und bezahlt werden, da weltweit rund 800 Millionen Menschen das Event live verfolgen (Mittag, 2020). Auch die Olympischen Spiele zählen zu den sogenannten Megaevents des Sports (Preuß, 1999; Andreff \& Weitzmann, 2019). Allein die Eröffnungszeremonien der Olympischen Sommerspiele in Peking, London und Rio wurden weltweit von einem Milliardenpublikum an den Bildschirmen verfolgt.

Bei der Vergabe der Sportgroßveranstaltungen zeichnete sich mit dem Beginn des 21. Jahrhunderts eine deutliche geografische Verlagerung ab. Während im 20. Jahrhundert die Austragung von Sportgroßereignissen zumeist großen OECD-Staaten wie etwa Deutschland und Frankreich oder Japan und den Vereinigten Staaten zugesprochen wurde, erfolgte die Vergabe von Sportgroßveranstaltungen im 21. Jahrhundert vor allem an BRICS-Staaten. Dies gilt namentlich für Olympische Spiele und Fußballweltmeisterschaften wie die Sport-Megaevents in Brasilien (Olympische Sommerspiele 2016, Fußballweltmeisterschaft 2014), in Russland (Olympische Winterspiele 2014, Fußballweltmeisterschaft 2018), in China (Olympische Sommerspiele 2008, Olympische Winterspiele 2022) und in Südafrika (Fußballweltmeisterschaft 2010) verdeutlichen. Zurückgeführt werden kann die veränderte Vergabepolitik darauf, dass die Staaten der OECD-Welt mittlerweile als weitgehend erschlossene bzw. gesättigte Sportmärkte gelten, während den BRICS-Staaten noch erhebliches Wachstumspotenzial zugeschrieben wird. Zugleich sind die Anforderungen an Olympische Spiele derart stark gestiegen, dass die traditionellen Ausrichternationen der westlichen Welt zunehmend weniger bereit sind, die immensen Veranstaltungskosten aufzubringen. Dies gilt umso mehr, da hier die Bevölkerung potenzieller Austragungsorte in den beiden vergangenen Dekaden verstärkt darauf gedrängt hat, in die Entscheidung einbezogen zu werden. In den in diesem Zusammenhang abgehaltenen Referenden sprach sich - wie in Hamburg, München und GarmischPartenkirchen - jedoch wiederholt eine Mehrheit gegen Olympische Spiele in der Heimatstadt aus (Nieland, Ihle \& Mittag, 2016).

Seitens der Kandidatenstaaten wird als Motiv für die Bewerbung um ein Sportgroßevent im Allgemeinen ein potenzieller wirtschaftlicher Mehrwert hervorgehoben. Erhofft werden von der Ausrichtung des Events neue Arbeitsplätze, ein Ausbau der Infrastruktur und eine Steigerung der Tourismuszahlen. Mit der Ausrichtung durch die BRICSStaaten sind aber weitere Motive hinzugekommen. Für diese stellen die eigenen globalen Präsentationsmöglichkeiten durch das Event, verbunden mit einer erhofften Zunahme an Bekanntheit im Sinne des "Nation Branding[s]" (Nauright, 2013; Kobierecki \& Strożek, 2017) sowie ein potenzieller Imagegewinn (Gripsrud, Nes \& Olsson, 2010), ebenfalls 
zentrale Motive dar. Hinzu kommt, dass Sportgroßveranstaltungen auch verstärkt als Instrument eingesetzt werden, das im Sinne der Popularitäts- und Legitimationssteigerung von Regierungen oder Verbänden die nationale Öffentlichkeit beeindrucken soll. Darüber hinaus wird SportMegaevents auch eine identitätsstiftende Wirkung zugeschrieben. Mit dieser Ausrichtung und mit diesen Zielsetzungen haben sich Sportgroßereignisse zu einer "globale[n] Bühne“ der Politik entwickelt (Mittag \& Nieland, 2012). Da das IOC gewissermaßen durch die Wahl der Ausrichterstädte das Fundament für diese politische Bühne bereitet hat, kann es auch nicht verwundern, wenn die Olympischen Spiele ihren Charakter als primär sportlicher Wettbewerb verlieren und damit mittelbar auch die Autonomie des IOC berührt wird.

Der Umstand, dass die Olympischen Spiele zunehmend zum Spielball der Interessen geworden sind, hat nicht zuletzt auch zu den zahlreichen sportbezogenen Protestaktivitäten der vergangenen Jahre geführt. Diese reichten von Demonstrationen und Störungen beim Fackellauf im Vorfeld der Olympischen Spiele in Peking bis zum Entschluss hochrangiger Politiker des Westens, darunter Staats- und Regierungschefs von Frankreich, Großbritannien, Deutschland, Kanada und den USA, den Olympischen Winterspielen in Sotschi keinen Besuch abzustatten. Unübersehbar ist, dass mit politischer Kritik am Austragungsort auch Kritik an der Olympischen Bewegung verbunden ist. Dies gilt vor allem mit Blick auf die sozialen, humanitären und ökologischen Rahmenbedingungen von Sportgroßereignissen. Da nicht wenige der wachstumsstarken Staaten (noch) keine stabilen Demokratien sind, besitzen Kriterien wie Menschenrechte oder Umweltstandards in diesen Ländern einen eher geringen Stellenwert. So erfolgten in Brasilien im Zuge der Fußballweltmeisterschaft und der Olympischen Spiele zahlreiche Enteignungen und Zwangsumsiedlungen (Mittag \& Ebbinghaus, 2017). Schätzungen zufolge waren allein in Rio de Janeiro 22 Armenviertel von Abrissarbeiten betroffen, so dass rund 65.000 Favela-Bewohner*innen ihr angestammtes Heim aufgeben mussten. Für die Bewohner bedeuteten die Zwangsumsiedlungen nicht nur den Verlust ihres Wohnraums, sondern bisweilen auch eine soziale Entwurzelung. Vor allem von den Medien und von Menschenrechtsorganisation wird infolgedessen im Zuge des Bewerbungsverfahrens verstärkt auf die Menschenrechtsproblematik verwiesen, womit der Entscheidungsautonomie des IOC über den Austragungsort aber auch Grenzen aufgezeigt werden. Dies gilt umso stärker, weil die Gegenargumente der Olympischen Bewegungen nicht überzeugen konnten. Im Zuge des Vergabeprozesses der Sommerspiele 2008 an China hatte man im Umfeld der Olympischen Bewegung noch darauf verwiesen, dass der 
sportliche Rahmen auch zu einer Verbesserung der Arbeits- und Lebensverhältnisse sowie der demokratischen Standards beitragen könne. Nach den Spielen fiel das Urteil allerdings ernüchternd aus. Die Erwartung, dass sich die Menschenrechtssituation in China durch die Olympischen Spiele 2008 mittelfristig bessern würde, blieb Wunschdenken. Zahlreiche Oppositionelle waren während der Spiele unter Hausarrest gestellt oder in Haft genommen worden, zugleich wurden die Medien streng zensiert. Selbst die Kommunikation via Internet war während der Olympischen Spiele nur eingeschränkt möglich (Kidd, 2010).

Dass die Sportgroßereignisse gerade in den BRICS-Staaten zu einer Bühne des Protests geworden sind, dokumentieren auch die Proteste der Athlet*innen. So hatte ein halbes Jahr vor den Winterspielen in Sotschi die schwedische Weltklasse-Hochspringerin Emma Green bei der Leichtathletikweltmeisterschaft in Moskau im August 2013 in der Qualifikation ihre Fingernägel in den Regenbogenfarben lackiert. Diese eher subtile Demonstration von Protest war als Ausdruck gegen die Diskriminierung von Homosexuellen in Russland zu verstehen, mit dem die Schwedin auf die seinerzeitigen politischen Entwicklungen um das russische AntiHomosexuellen-Gesetz im Austragungsland der bevorstehenden Olympischen Winterspiele aufmerksam machen wollte (Mittag, 2016). Entsprechender demonstrativer Protest ist zumeist ohne größeren Aufwand und vergleichsweise spontan umzusetzen. Er besitzt hohes symbolisches Potenzial, er ist gut sichtbar und die Medien können ihn gut vermitteln. Dies umso mehr, da im Zeitalter der sozialen Netzwerke keine Geste unbeachtet bleibt und die politischen Rahmenbedingungen nicht mehr einfach mit dem Hinweis auf die vermeintlich unpolitische Dimension des Sports ausgeblendet werden können.

Das IOC hat unter der Präsidentschaft von Thomas Bach auf diese in erheblichem Maße selbst verursachten Protestaktivitäten reagiert. Einen Meilenstein markiert dabei die Agenda 2020, die von Thomas Bach im Jahre 2014 als umfassendes Reformwerk der Olympischen Bewegung vorgestellt wurde. Unter der Parole, „das IOC müsse sich wandeln, um nicht zum Spielball der wirtschaftlichen und gesellschaftlichen Entwicklung zu werden" (Die Zeit online, 8.12.2014) hatte Thomas Bach unter anderem angeregt, zukünftig mehr auf Nachhaltigkeit zu setzen und wieder mehr Bewerberstädte für Olympische Spiele zu gewinnen - nicht zuletzt Staaten aus etablierten Demokratien. Damit sollte verhindert werden, dass die Kosten für Olympische Spiele ins Gigantische stiegen und sie zudem weiterhin für Propagandazwecke instrumentalisiert werden. Diese Zielsetzung ging bei den Bewerbungen für 2024 und 2028 zumindest in Teilen auf. Es traten zunächst zwar zahlreiche westliche Metro- 
polen an, dann zogen Boston, Hamburg, Rom und Budapest ihre Bewerbungen primär aus Sorge vor zu hohen Kosten jedoch zurück. Am Ende konnten mit Paris und Los Angeles dann aber doch zwei Austragungsstädte aus demokratischen Staaten gekürt werden.

Unter dem Titel Agenda 2020+5 wurde der Reformprozess von Thomas Bach im Jahr 2021 erneut aufgegriffen. Zu den aktualisierten Zielsetzungen zählte die „Stärkung der Rolle des Sports als Wegbereiter für UNZiele bezüglich von Nachhaltigkeit“ oder die „Unterstützung für Flüchtlinge und Bevölkerungsgruppen, die von Vertreibungen betroffen sind“. Mit diesen Zielen dokumentiert das IOC sein Bewusstsein für gesellschaftliche Problemfelder, die auch den Sport berühren. Zugleich nimmt das IOC aber in Kauf, weiterhin zum Gegenstand von politischen Debatten zu werden.

\section{Der Rekurs auf die Sportdiplomatie}

Eine ähnliche Tendenz zeigt sich auch im letzten hier behandelten Problemfeld: der Sportdiplomatie. Die Nutzung des Sports als Instrument der internationalen Politik ist keine neue Entwicklung, sondern wurde schon im frühen 20. Jahrhundert als probates Mittel vor allem der auswärtigen Politik von Nationalstaaten genutzt. Unter den analytischen Kategorien Sportdiplomatie und Soft Power hat diese spezifische Form internationaler Politik in den beiden letzten Dekaden aber mittlerweile verstärkte Beachtung gefunden. Politische Beobachter machen sogar eine systematische Nutzung aus, wenn etwa die Europäische Kommission im Jahr 2016 eine eigene Arbeitsgruppe zum Thema Sportdiplomatie einrichtet, die darauf zielt, das politische Potenzial des Sports auch für internationale Organisationen bzw. supranationale Gemeinschaften wie die Europäische Union zu beleuchten. Eine ähnliche Tendenz ist auch in der Olympischen Bewegung auszumachen. In den beiden letzten Dekaden ist die Sportdiplomatie auch seitens des IOC mit Blick auf die Olympischen Spiele in neue Dimensionen vorgestoßen, wie vor allem das Beispiel Korea dokumentiert.

Nachdem die Entspannungsprozesse zwischen Nord- und Südkorea jahrzehntelang brachlagen, ist es im Vorfeld der Olympischen Winterspiele im südkoreanischen Pyeongchang 2018 zu einer neuerlichen Annäherung beider Staaten gekommen. Der gemeinsame Einzug der Athlet*innen aus Nord- und Südkorea bei der Eröffnungsfeier sowie das gemeinsame Eishockeyteam der Frauen waren Elemente einer symbolischen Politik von Präsident Moon Jae-in und Machthaber Kim Jong-un, 
die vom IOC nicht nur begrüßt, sondern offensiv unterstützt wurden. Bei der Abschlussfeier der Winterspiele 2018 von Pyeongchang erklärte IOCPräsident Thomas Bach: „Ihr habt gezeigt, dass der Sport die Menschen in einer fragilen Welt zusammenbringen kann. Ihr habt gezeigt, wie der Sport Brücken bauen kann“ (Deutsche Welle, 2019). Angesichts des Erfolgs dieser Initiative bei den Olympischen Winterspielen wurden im Jahr darauf sogar Pläne geschmiedet, dass Nord- und Südkorea in mehreren Sportarten mit gemeinsamen Mannschaften bei den Olympischen und Paralympischen Spielen in Tokio antreten. Diese Idee fand erneut die Zustimmung des IOC, das sich bereit erklärte, das Vorhaben begleitend zu unterstützen. Wohlwollend unterstützt wurde auch die Entscheidung der beiden Frauenmannschaften aus Nord- und Südkorea, bei der Tischtennis-WM in schwedischen Halmstad im April 2018 nicht im Viertelfinale gegeneinander anzutreten, sondern vielmehr im Halbfinale ein gemeinsames koreanisches Team zu formieren. Als sich im Februar 2019 Vertreter Nord- und Südkoreas mit Thomas Bach in Lausanne trafen, um nähere Details zu erörtern, nahm der IOC-Präsident erneut auf die Brückenbau-Metapher Bezug und betonte die Chancen der Zusammenarbeit. In diesem Zusammenhang begrüßte der IOC-Präsident sogar eine potenzielle gemeinsame Bewerbung beider Länder für die Olympischen Sommerspiele 2032.

Das Beispiel Korea zeigt, dass Sport Versöhnung und Frieden befördern kann. Allerdings sind auch die Grenzen des Ansatzes nicht zu übersehen. Im Sommer 2020 beendete Nordkorea die Phase des Tauwetters und brach die diplomatischen Beziehungen zu Südkorea wieder ab. Wie realitätsnah entsprechende Pläne sind und ob sich der organisierte Sport hier nicht sogar angreifbar macht, bleibt abzuwarten. Die Türöffner-Funktion des Sports kann durchaus auf Erfolge zurückschauen, aber der Grat bei Verhandlung mit autoritären Regimen oder Diktaturen ist schmal. Infolgedessen wird in sportpolitischen Beiträgen auch wiederholt darauf verwiesen, dass die in den letzten Jahren angestoßene Debatte über Werte im Sport, in der auch das Ideal der Autonomie des Sports als Leitbild hochgehalten wird, nicht zugunsten der Sportdiplomatie geopfert werden sollte.

\section{Perspektiven und Grenzen des Leitbilds der Autonomie im olympischen Sport}

Die Ausführungen dieses Beitrages haben angedeutet, wie stark sich die Welt des Sports in den vergangenen Jahren verändert hat. Zugleich haben sie Beispiele dafür geliefert, in welchem erheblichen Ausmaß der olympi- 
sche Sport und die Olympische Bewegung zum Bestandteil politischer Prozesse geworden sind. In den vergangenen Jahren hat sich zunehmend deutlicher die Herausbildung eines eigenen Politikfelds Sport herauskristallisiert, das durch zahlreiche Wechselwirkungen gekennzeichnet ist. Entsprechende Verflechtungen hat es zwar in der Sportgeschichte schon immer gegeben - von Hitlers Olympioniken bis zu dem auch im Sport ausgetragenen Systemwettbewerb im Kalten Krieg - aber erst mit der Ökonomisierung und Medialisierung des Sports in den letzten Dekaden und der damit einhergehenden Mobilisierung und Interessendifferenzierung hat die Sportpolitik deutlichere Konturen gewonnen. Sport stellt damit nicht länger mehr nur ein Tätigkeitssystem dar, das primär durch sportbezogene Regeln gestaltet wird. Vielmehr markiert der Sport durch die Verflechtung mit anderen Politikfeldern, Interessen und Akteuren auch zunehmend ein Konfliktfeld, in dem es um die Durchsetzung von Interessen sowie die Herstellung von Entscheidungen geht.

In einer derartigen Konstellation kann es eine Autonomie des olympischen Sports im engeren Sinne aber nicht mehr geben. Die Olympische Bewegung ist sowohl Treiber als auch Getriebener in einem komplexen Zusammenspiel von Akteuren, das eine umfassende Selbstdefinition, Selbstorganisation und Selbstregulierung durch den Sport allein nicht mehr erlaubt. Im Umkehrschluss bedeutet dies aber auch nicht, dass der Sport um jeden Preis zu politisieren ist. Vielmehr bedarf es einer eingehenderen Befassung mit der künftigen Auslegung von Autonomie im Rahmen der Olympischen Idee unter den veränderten Vorzeichen des 21. Jahrhunderts. Ein eingehender Diskurs über die sportpolitische Dimension von Autonomie steht aber noch aus. Infolgedessen spricht einiges dafür, die Sportpolitik zu einem wichtigen Baustein der sportlichen Bildung zu erheben. Ein erster Schritt könnte dabei in einer grundlegenden Bestandsaufnahme bestehen, die systematischer als der hier vorgelegte kursorische Überblick die sportpolitischen Entwicklungen, Akteure und Konflikte der letzten Jahre erfasst. Auf diesem Fundament aufbauend könnten dann in offenen Foren eine eingehendere gesellschaftliche Debatte über Ziele, Leitbilder und Methoden geführt werden, die Denkanstöße und Perspektiven für die Ausgestaltung der Olympischen Idee und der Zukunft des Sports eröffnet. 


\section{Literatur:}

Bach, T. (2013). Statement on the occasion of the adoption of the resolution "Building a peaceful and better world through sport and the Olympic ideal". Speech delivered to the $68^{\text {th }}$ Session of the United Nations General Assembly in New York, 6.11.2013.

Bonollo De Zwart, F. \& Gilligan, G. (2009). Sustainable governance in sporting organisations In P. Rodriguez, S. Késenne \& H. Dietl (Hrsg.), Social responsibility and sustainability in sports (S. 165-227). Oviedo: Universitat de Oviedo.

Bette, K.-H. (1990). Sport als Thema geselliger Konversation. Zur Choreographie mikrosozialer Situationen. In W. Kleine \& W. Fritsch (Hrsg.), Sport und Geselligkeit. Beiträge zu einer Theorie von Geselligkeit im Sport (S.61-80). Aachen: Meyer \& Meyer.

Chappelet, J.-L. \& Kübler-Mabbott, B. (2008). The International Olympic Committee and the Olympic system: The Governance of World Sport. London: Routledge.

Chappelet, J.-L. (2010). The autonomy of sport in Europe. Strasbourg: Council of Europe.

Chappelet, J.-L. (2018). The autonomy of sport and the EU. In J. Anderson, R. Parrish, \& B. García (Hrsg.), Research handbook on EU sports law and policy (S. 157-172). Cheltenham: Edward Elgar.

Cotterell, M. \& Vöpel, H. (2020). Sport und Ökonomie. Ein Ambivalentes Verhältnis. In Zeitschrift für Politik und Gesellschaft, 9, 69-76.

Deutsche Welle online (2019). Korea: Wie der Sport Brücken baut, 14.2.2019.

Die Zeit online (2014). IOC beschließt historische Reformen, 8.12.2014.

Emrich, E. \& Gassmann, F. (2019). Korruption und Sport. In P. Graeff \& T. Rabl (Hrsg.), Was ist Korruption? Begriffe, Grundlagen und Perspektiven gesellschaftswissenschaftlicher Korruptionsforschung, 2. Auf. (S. 143-164). Nomos.

Europäische Kommission (2007). White Paper on Sport. 11 July 2007, (COM 2007) 391 final.

Europäische Kommission (2011). Communication from the Commission to the European Parliament, the Council, the European Economic and Social Committee and the Committee of the Regions: Developing the European Dimension in Sport. 18 January 2011, (COM 2011) 12 final.

Expert Group Good Governance (2013). EU work plan for sport 2011-2014. Deliverable 2. Principles of good governance in sport. Abgerufen am 20. April 2021 unter https://ec.europa.eu/assets/eac/ sport/library/policy_documents/xg-gg-201307dlvrbl2-sept2013.pdf

Geeraert, A. (2013). The governance agenda and its relevance for sport: introducing the four dimensions of the AGGIS sports governance observer. In J. Alm (Hrsg.), Action for Good Governance in International Sports Organisations. Final report (S. 9-21). Kopenhagen: Play the game/Danish Institute for Sport Studies. 
Geeraert, A., Mrkonjic, M., \& Chappelet, J-L. (2015). A rationalist perspective on the autonomy of international sport governing bodies: Towards a pragmatic autonomy in the steering of sports. International Journal of Sport Policy and Politics, 7 (4), 473-488.

Gripsrud, G., Nes, E. B. \& Olsson, U. H. (2010). Effects of Hosting a Mega-Sport Event on Country Image. Event Management 14, 193-204.

Hallmann, K. \& Petry, K. (Hrsg.) (2013): Comparative Sport Development. Systems, Participation and Public Policy, New York: Springer Verlag.

Haug, T. (2006). Doping. Dilemma des Leistungssports. Hamburg: Merus-Verlag.

International Olympic Committee/Comité International Olympique (1908). Annuaire [Lausanne].

International Olympic Committee/Comité International Olympique (1949). Olympic Rules/Charter of the Olympic Games. Lausanne.

International Olympic Committee/Comité International Olympique (1955). The Olympic Games: Charter, Rules and Regulations, General information. Lausanne.

International Olympic Committee (1979). Olympic Charter. Lausanne.

International Olympic Committee (2008). Basic universal principles of good governance of the Olympic and sports movement. Lausanne.

International Olympic Committee (2020). Olympic Charter. Lausanne.

Kidd, B. (2010). Human rights and the Olympic Movement after Beijing. Sport in Society. Cultures, Commerce, Media, Politics 13, 901-910.

Kleinfeld, R. (2018). Die europäische Sportpolitik und die Frage „Who Governs?“. Politikwissenschaftliche Zugänge. In J. Mittag (Hrsg.). Europäische Sportpolitik: Zugänge, Akteure, Problemfelder, (S. 65-88). Baden-Baden: Nomos Verlag.

Kobierecki, M. M. \& Strożek, P. (2017). Sport as a Factor of Nation Branding: A Quantitative Approach. The International Journal of the History of Sport 7 (8), 697-712.

Krieger, J. (2018). Manipulation in athletics: Historical and contemporary ties between on- and off-field corruption in the International Association of Athletics Federations (IAAF). The International Journal of the History of Sport, 35 (2-3), 231-246.

Krieger, J. \& Wassong, S. (2021). The Composition of the IOC: Origins and key turning points in the governance of the Olympic movement. In D. Chatziefstathiou/B. García \& B. Séguin (Hrsg.). Routledge Handbook of the Olympic and Paralympic Games (S. 203-216). Berlin, New York, London: Routledge.

Lennartz, K. (2009): Olympische Boykotte. In A. Bruns \& W. Buss (Hrsg.), Sportgeschichte erforschen und vermitteln. Jahrestagung der dvs-Sektion Sportgeschichte vom 19.-21. Juni 2008 in Göttingen (S. 179-200). Hamburg.

MacAloon, J. J. (2011). Scandal and governance: inside and outside the IOC 2000 Commission. Sport in Society, 14 (3), 292-308.

Mason, D.S., Thibault, L. \& Misener, L. (2006). An agency theory perspective on corruption in sport: The case of the International Olympic Committee. Journal of Sport Management, 20 (1), 52-72. 
Meier, E. (2021). Political autonomy and control of national Olympic committees. In D. Chatziefstathiou, B. García \& B. Séguin (Hrsg.). Routledge Handbook of the Olympic and Paralympic Games (S.265-274). Berlin, New York, London: Routledge.

Mittag, J. (2010). Die Entdeckung der Sportpolitik: Von der verbandlichen Autonomie zum Politikfeld Sport. Der Hammelsprung 1, 32-35.

Mittag, J. \& Nieland, J.-U. (2012). Die globale Bühne: Sportgroßereignisse im Spannungsfeld von politischer Inszenierung und demokratischen Reformimpulsen. Zeitschrift für Politikwissenschaft, 21 (4), 623-632.

Mittag, J. \& Nieland, J.-U. (2016). Lizenz zum Machterhalt: Das System FIFA und die Grenzen von Protest und Opposition in internationalen Sportorganisationen. Zeitschrift für Politikwissenschaft 2, 197-216.

Mittag, J. (2016). Bühne des Protests. In W. Billows \& S. Körner (Red.): Kulturreport: Globales Spiel - Sport, Kultur, Entwicklung und Außenpolitik, Göttingen: EUNIC Jahrbuch 2016, S. 180-188.

Mittag, J. \& J. Ebbinghaus (2017). „Juntos num só ritmo"? Brasilien und FIFA im Spannungsfeld der Interessen um die Vorbereitung und Vergabe der Weltmeisterschaft 2014. In H. Ihle et al. (Hrsg.): Globales Mega-Event und nationaler Konfliktherd. Die Fußball-WM 2014 in Medien und Politik (135-154). Wiesbaden: VS Verlag.

Mittag, J. (2018). Europäische Sportpolitik: Zugänge, Akteure, Problemfelder. BadenBaden: Nomos Verlag.

Mittag, J. (2019). Sportpolitik, in: W. Weidenfeld/W. Wessels (Hrsg.): Jabrbuch der Europäischen Integration 2019 (279-280). Baden-Baden: Nomos Verlag.

Mittag, J. (2020). Sportgroßveranstaltungen in Schwellen- und Entwicklungsländern: Profilsuche im Spannungsfeld von Erwartungen und Ernüchterungen. In K. Petry (Hrsg.), Sport im Kontext von internationaler Zusammenarbeit und Entwicklung. Perspektiven und Herausforderungen im Spannungsfeld von Wissenschaft, Politik und Praxis (91-102). Opladen/Berlin/Toronto: Barbara Budrich.

Nauright, J. (2013). Selling Nations to the World through Sports: Mega Events and Nation Branding as Global Diplomacy. Public Diplomacy Magazine 9, 22-27.

Nieland, J.-U., Ihlen, H. \& Mittag, J. (2016). Sportorganisationen unter Beobachtung: Die Olympiabewerbung Münchens 2018 in der Berichterstattung. In A. Hebbel-Seeger/T. Horky/H.-J. Schulke (Hrsg.): Sport als Bühne. Mediatisierung von Sport und Sportgroßveranstaltungen (232-257). Aachen. Meyer und Meyer.

Preuß, H. (1999). Ökonomische Implikationen der Ausrichtung Olympischer Spiele von München 1972 bis Atlanta 1996. Kassel: Agon-Sportverlag.

Preuß, H., Andreff, W. \& Weitzmann M. (2019). Cost and Revenue Overruns of the Olympic Games 2000-2018. Wiesbaden: Gabler Verlag.

Risse, G. (2018). Funktionale Institutionen-Bildung in der Anti-Dopingpolitik der Bundesrepublik Deutschland. Akteureinflüsse, Akteurkonstellationen, Akteurinteraktionen. Köln: DSHS Köln.

Schümer, D. (1996). Gott ist rund. Die Kultur des Fußballs. Berlin: Suhrkamp. 
Sobry, C. (2011). Sports Governance in the World: A Socio-Historic Approach. Paris: Éd. Le Manuscrit.

United Nations (2014). General Assembly. Sixty-ninth session. $28^{\text {th }}$ plenary meeting. New York.

Vedder, C. \& Lämmer, M. (2014). Olympische Charta 2014. Frankfurt am Main: Bernecker. 
